\title{
Determinantes de gastos en salud durante el último año de vida de la población mexicana de 50 años 0 más
}

\author{
Determinants of health expenditures during the last year of life \\ of the Mexican population aged 50 or over
}

\author{
Jesús Daniel Zazueta-Borboa y Roberto Ham-Chande \\ Netherlands Interdisciplinary Demographic Institute, Holanda y Departamento \\ de Estudio de Población del El Colegio de la Frontera Norte, México
}

\section{Resumen}

En las edades mayores el último año de vida es cuando la salud más se deteriora y es la etapa más demandante de atención médica y de cuidados. En consecuencia, también es la etapa más costosa. Se analizan diferencias por sexo en los determinantes de los gastos en salud por cuenta propia durante el último año de vida de personas de 50 o más, usando la encuesta del Estudio Nacional de Salud y Envejecimiento en México. Con estimaciones de gasto en tres niveles: ninguno, medio-bajo, y alto, controlando por variables sociodemográficas los resultados indican que el número de noches de hospital es la mayor determinante de gastos en salud, por encima de los relacionados con las enfermedades reconocidas como causa de muerte. Este factor tiene implicaciones en los gastos tanto por cuenta propia y como de las instituciones médicas. Son temas relevantes ante los cambios en el sistema de salud pública.

Palabras clave: Envejecimiento; último año de vida; gastos en salud, pagos por cuenta propia.

Abstract

During the last year of life of the elderly health deteriorates the most, thus becoming the period of time when needs of medical services, personal care requirements and related costs are at highest. Using the Mexican Health and Aging Study, differences by sex in the determinants for out-ofpocket health expenditures during the last year of life of people 50 and over are discussed. Three levels of out-of-pocket expenses disbursement are contemplated: none, medium-low, and high. Controlling by sociodemographic variables, findings suggest that the number of hospital nights is the main determinant of health expenses, even above those related to diseases that are regarded as main causes of death. This fact impacts both out-of-pocket expences and the costs for medical institution. It is another issue in the over-hauling of the public health system.

Keywords: Aging, last year of life, health costs, out-of-pocket expences. 


\section{INTRODUCCIÓN}

$\mathbf{S}$ e ha documentado cómo y por qué durante el último año de vida (UAV) el estado de salud se deteriora y los gastos en salud se incrementan (Verbrudgge, 2016). Son estudios que han señalado la relación entre las enfermedades como causa de muerte y el monto del gasto en salud (Hogan et al., 2001). También señalan las diferentes trayectorias de salud y enfermedad y su influencia en los gastos (Aldridge et al., 2015). La mayor parte de las investigaciones se ha realizado en países europeos y Estados Unidos, que cuentan con acceso a registros administrativos de alta calidad, encuestas longitudinales y datos de aseguradoras privadas, permitiendo medir el incremento en los gastos en salud durante el UAV para evidenciar cómo el costo por salud en la vejez se concentra al final de la vida. Resulta relevante mostrar cuales son los retos económicos, las presiones al sistema de salud, y la participación de familias y hogares. A su vez, en países europeos se han encontrado variaciones en los gastos en salud por cuenta propial dependiendo del sistema de salud, el lugar de muerte y el porcentaje del PIB destinado a la atención de la salud (Orlovic et al., 2017; Penders et al., 2017).

Estas investigaciones hacen suponer que el proceso de envejecimiento de México tendrá contextos similares, parte de lo cual lo corroboran estudios descriptivos sobre las condiciones de salud, económicas, de género y familiares en el UAV (González et al., 2018) y los gastos por cuenta propia que se generan. Como factores relevantes aparecen las enfermedades que son causa principal de la muerte y los tipos de afiliación a servicios de salud (Salinas et al., 2019). Sin embargo, ante el ritmo de envejecimiento, la estratificación del sistema de salud y el efecto económico sobre hogares con algún miembro adulto mayor, conviene ampliar las preguntas sobre las consecuencias económicas, sociales y familiares durante el UAV. Se toma en cuenta que el sistema de salud se compone por diferentes servicios públicos y privados. Los servicios públicos en su mayoría son por afiliación a la seguridad social a través de un trabajo en el mercado laboral formal, además de instituciones de protección social.

A diferencia de países europeos donde la sanidad pública universal es amplia y de calidad, en América Latina es limitada y de baja eficiencia, de manera que hay necesidad de solventar los gastos de salud hasta donde

\footnotetext{
${ }^{1}$ Los gastos que se hacen mediante los propios recursos individuales o familiares siempre se han considerado ser "por cuenta propia". Pero existe ahora una tendencia a llamarlos "gastos de bolsillo" derivado del término inglés "pocket expenses" o "pocket money". En este artículo mantenemos el término castellano original de "cuenta propia".
} 
los recursos propios lo permitan. Esto se ilustra en la Figura 1, donde se compara el porcentaje del gasto por cuenta propia con respecto al gasto total en salud en México, en países de la Organización para la Cooperación y el Desarrollo Económicos (OCDE), en la Unión Europea y en América Latina y el Caribe. Destacan, claramente, las altas cifras de México.

Figura 1: Gastos por cuenta propia como porcentaje del gasto total en salud en México, América Latina, países de la OCDE y la Unión Europea

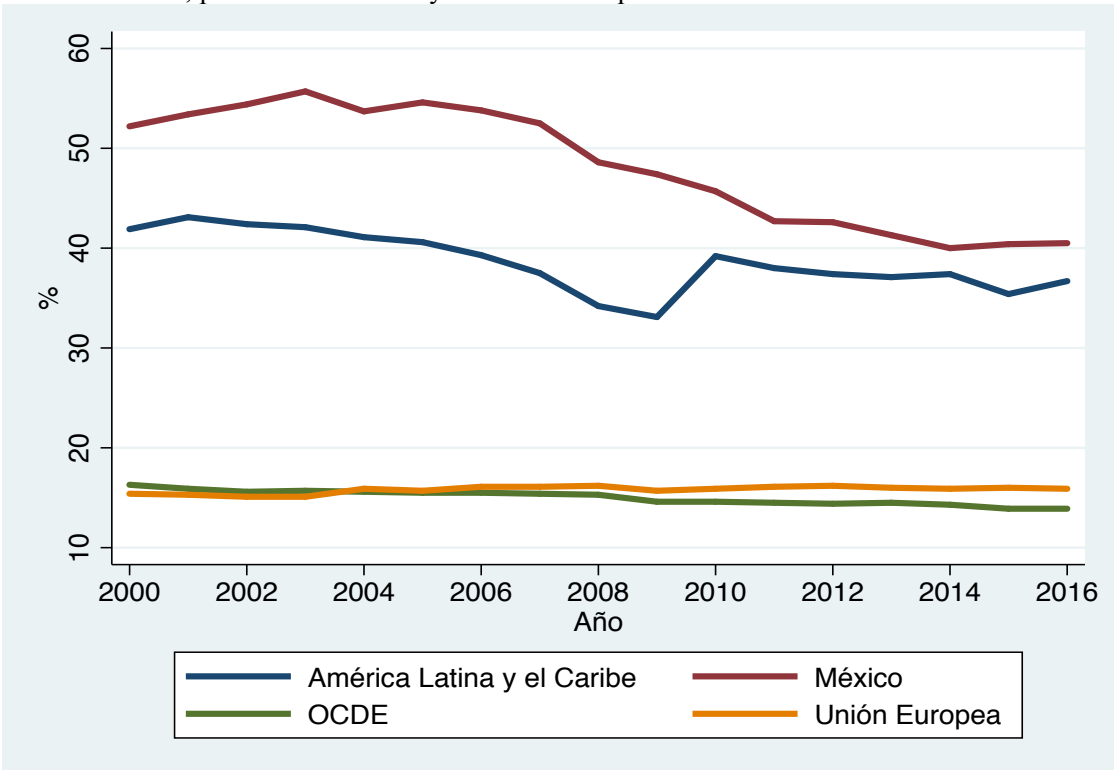

Fuente: elaboración propia con datos del Banco Mundial.

La falta de protección financiera es el principal determinante de los gastos por cuenta propia en América Latina (Knaul et al., 2001), con consecuencias económicas para los hogares en el corto y mediano plazo. Asimismo, hogares con miembros en edades mayores son más propensos a experimentar gastos catastróficos. ${ }^{2}$ Con la Encuesta Nacional de Ingresos y Gastos de los Hogares en México (ENIGH) se estima que en hogares con población envejecida hay 50 por ciento de más en el gasto en salud (Ceballos-Mina, 2019). Usando la misma encuesta, la presencia de gasto catastrófico es 4.2 por ciento superior en hogares con personas de 65

\footnotetext{
${ }^{2}$ La Organización Mundial de la Salud define gasto catastrófico en salud, aquellos gastos que hacen las personas en atención en salud y representan una cantidad mayor al 30 por ciento o más de su capacidad de pago (WHO, 2005).
} 
años o más, determinado por el deterioro de la salud después de esa edad (Granados y Nava, 2019).

Dado que el deterioro del estado de salud durante el UAV incrementa la necesidad y el costo de la atención médica, el objetivo de este artículo es analizar los gastos en salud en tres niveles: i) no incurrir en ellos; ii) que sean de rango bajo-medio; iii) que sea mayores, usando el Estudio Nacional de Salud y Envejecimiento en México (ENASEM). Una de las hipótesis que guían esta parte de la investigación es que el lugar de atención de salud es el principal determinante del nivel del gasto. Hasta ahora el estudio de los determinantes del gasto en salud ha considerado al hogar como unidad de análisis, sin estudiar diferencias entre sus integrantes. Bajo las premisas de que el deterioro del estado de salud durante el UAV incrementa la necesidad y el costo de la atención médica y que en el envejecimiento las condiciones de salud tienen más gravedad para las mujeres, un objetivo es analizar las condiciones en el UAV para cada sexo en relación con los tres niveles de gasto.

Las siguientes secciones presentan, primero, la situación socioeconómica en la vejez de los hogares con personas adultas mayores junto con los principales determinantes del gasto en salud. Enseguida se exponen los datos y el abordaje metodológico sobre los determinantes del gasto en salud de los adultos mayores durante el UAV. Como tercera parte se muestran los resultados para finalizar en conclusiones y propuestas.

\section{CONDICIONES SOCIOECONÓMICAS DETERMINANTES}

\section{DEL GASTO EN SALUD}

La vejez y el envejecimiento se correlacionan con creciente vulnerabilidad y dependencia en tres categorías: seguridad económica, atención a la salud y cuidados personales (Ham, 2003). Estas clases de dependencia tienen dos conocidas particularidades a las que ahora se propone agregar una más. Las características más conocidas son por una parte la intensa interrelación que existe entre ellas y por otra que están condicionadas por factores socioeconómicos y de salud experimentadas en las etapas de vida antes del envejecimiento. La tercera y que ahora se propone y examina es cómo se agravan durante el UAV.

Reiterando la mayor longevidad de las mujeres, pero en peores condiciones de salud y funcionalidad que los hombres (Díaz et al., 2016; Díaz y Wong, 2016), se propone conocer cómo estas diferencias inciden en la atención médica, los cuidados y los gastos por cuenta propia. 
En este estudio es particularmente relevante observar el caso de las personas que fueron o son económicamente activas, donde las características del mercado laboral durante la etapa productiva determinan sustancialmente las condiciones en la vejez y en el UAV. Ya se ha mencionado que un factor principal de la salud y los gastos durante el UAV es el acceso a servicios sanitarios, condicionado por el estatus socioeconómico y las características del mercado laboral.

Asimismo, existen factores históricos a tomarse en cuenta. Las cohortes que en el siglo XXI ya están en la etapa de la vejez, vivieron grandes transformaciones en el siglo XX que influyeron en oportunidades sociales y laborales, incluyendo que los grupos asalariados y urbanos fueran las beneficiarias de la naciente y creciente seguridad social (Barba, 2013; Valencia, Foust y Tetreault, 2012). También experimentaron novedades y avances como la educación básica pública, laica y obligatoria, los sistemas públicos de salud, el sindicalismo, los créditos a la vivienda y la seguridad social.

La Tabla 1, elaborada con la primera encuesta de ENASEM, indica la proporción estimada de la población de 50 o más que en 2001 tenía prestaciones laborales ligadas a su empleo principal. ${ }^{3}$ Las cifras permiten inferir una correlación entre edad y beneficios recibidos en favor de las edades más jóvenes en todas las prestaciones. Una explicación viene al considerar los tiempos en la creación y desarrollo de las instituciones de apoyo y protección del empleo en el siglo XX. Se observan brechas entre hombres y mujeres en materia de derechos laborales, pero también tendencias a cerrar diferencias según se manifiesta en las edades más jóvenes, que denotan beneficios debida a la mayor participación femenina en el mercado laboral.

Sin embargo, se admite que, aunque se hayan extendido y mejorado las prestaciones laborales, los porcentajes de población cubierta y el nivel de beneficios aún no son completos, lo que también se nota en desigualdades que afectan al envejecimiento. Una consecuencia ya mencionada, es la presión sobre las familias al encargarse de la dependencia de parientes envejecidos, incluyendo los costos en salud y atención durante el UAV. De hecho, son muchas las familias que no cuentan con acceso a servicios adecuados de salud y cuyos familiares envejecidos pueden llevarlas a adversidades económicas.

\footnotetext{
3 Se consideró trabajo principal, utilizando la batería de preguntas donde se pide que diga las características del trabajo principal que desempeñó a lo largo de su vida, en el ENASEM 2001.
} 
Tabla 1: Porcentajes de la población de 50 o más por tipo de beneficios ligados al trabajo principal, sexo y grupos de edad durante su vida laboral

\begin{tabular}{lrrrrrr}
\hline \multicolumn{1}{c}{ Prestación } & \multicolumn{7}{c}{ Grupos de edad } \\
& 50 a 59 & 60 a 69 & 70 a 79 & 80 a 89 & 90 o más & Total \\
\hline Hombres & & & & & & \\
IMSS (1) & 50.0 & 53.5 & 50.6 & 40.2 & 35.7 & 50.7 \\
ISSSTE (2) & 13.7 & 9.6 & 8.6 & 13.4 & 14.3 & 11.7 \\
SAR (3) & 35.5 & 24.9 & 12.9 & 11.3 & 7.1 & 27.5 \\
AFORE (4) & 28.5 & 19.3 & 6.6 & 5.2 & 0 & 21.1 \\
Crédito de vivienda & 40.1 & 30.9 & 22.3 & 20.6 & 14.3 & 33.5 \\
Seguro médico privado & 10.2 & 7.9 & 10.1 & 6.2 & 14.3 & 9.4 \\
Seguro de vida & 28.3 & 22.6 & 16.5 & 14.4 & 7.1 & 24.0 \\
Mujeres & & & & & & \\
IMSS (1) & 28.8 & 22.2 & 12.6 & 7.5 & 0 & 23.7 \\
ISSSTE (2) & 9.8 & 6.8 & 5.6 & 5.0 & 8.3 & 8.2 \\
SAR (3) & 16.5 & 8.7 & 4.9 & 3.8 & 0 & 12.1 \\
AFORE (4) & 12.3 & 5.8 & 2.5 & 1.3 & 0 & 8.6 \\
Crédito de vivienda & 19.6 & 11.9 & 8.1 & 3.8 & 8.3 & 15.1 \\
Seguro médico privado & 4.9 & 5.5 & 1.8 & 3.8 & 8.3 & 4.6 \\
Seguro de vida & 12.3 & 8.4 & 5.3 & 3.8 & 16.7 & 9.9 \\
\hline
\end{tabular}

(1) Instituto Mexicano del Seguro Social.

(2) Instituto de Seguridad y Servicios Sociales de los Trabajadores del Estado.

(3) Sistema de Ahorro de Retiro.

(4) Administradoras de Fondos para el Retiro.

Fuente: elaboración propia con base de ENASEM 2001.

Se ha intentado dilucidar si los gastos catastróficos pudieron subsanarse con el Sistema de Protección Social en Salud mejor conocido como Seguro Popular (SP), implantado en 2004 y cancelado en 2020. ${ }^{4}$ Este sistema ha desaparecido y se trataba de un organismo que no era proveedor y ofertante de atención y servicios de salud, sino que como seguro cubría el costo de servicios recibidos de parte de la estructura pública y privada previamente autorizada (Moreno y Flamand, 2015). Se señala que el SP tuvo un efecto positivo sobre la atención en salud de población envejecida, especialmente en áreas rurales (Parker, Saenz y Wong, 2017), además de reducción de gastos catastróficos (Knaul et al., 2018; Granados y Nava, 2019, Ceballos, 2019). Pero también se muestra que, si bien se amplió la cobertura en ser-

4 En 2020, el programa del Seguro Popular ya desapareció y se remplazó por el Instituto Nacional de Salud para el Bienestar (INSABI). 
vicios de salud, la calidad de los servicios fue deficiente, incluyendo difícil acceso y carencia de medicamentos (Luna, 2015) y sin mejora en la salud de la población envejecida (Moreno y Flamand, 2015; Barba, 2015).

Otras características del gasto durante el UAV incluyen que cada noche de hospital en cuidados intensivos es superior a veinte mil pesos, ${ }^{5}$ y es mayor para las personas que no tienen derecho a servicios de salud (González et al., 2018). Asimismo, se constata que el gasto varía según la causa principal de muerte (Salinas et al., 2019), que es creciente al compararlo con años previos, $\mathrm{y}$ también es mayor con lo erogado en salud por personas de los mismos grupos de edades que no fallecieron (Zazueta, 2018). Se agrega que cuando se cuenta con acceso a servicios de salud, el gasto por cuenta propia depende de la institución de afiliación (Zazueta, 2018; González et al., 2018; Salinas et al., 2019).

De esta manera, el acceso a servicios de salud da lugar a desigualdades en el gasto médico y de atención durante el UAV, aunque aún no se cuenta con un diagnóstico sobre la magnitud de las diferencias y la naturaleza de cada determinante. Aún se requiere afinar conceptos, crear definiciones, generar datos y adoptar métodos. En busca de guías de trabajo frente estas limitaciones se mencionan que en Europa los principales determinantes del gasto en el UAV están relacionados con las características del sistema de salud y las políticas de cuidados paliativos. A su vez estas características influyen en el lugar de la muerte, ya sea en hogar o en hospital, y éste último factor incide directa y mayormente en los gastos en salud. Por otra parte, la mayor parte del gasto por cuenta propia es para el cuidado personal (Orlovic et al., 2017; Penders et al., 2017).

\section{INFORMACIÓN E INSTRUMENTOS DE ANÁLISIS}

En este estudio la fuente principal de información es la base de datos del Estudio Nacional de Salud y Envejecimiento en México (ENASEM). Es una encuesta longitudinal tipo panel, representativa de la población de 50 años o más en México que cuenta con cuatro rondas, 2001, 2003, 2012 y 2015. Una virtud de esta encuesta es la baja atrición de los participantes del panel, en comparación con estudios similares (Orozco, Wong y Michaels, 2018: 71). En 2012 se añadieron más personas en el grupo de edad de 50 a 59 años para mantener la representatividad.

\footnotetext{
5 Ese cálculo fue para el año 2012 y la paridad del peso mexicano frente al dólar el 1 de junio de 2012 era un dólar por 14.41 pesos mexicanos, lo que da un valor de 1,428.5 dólares.
} 
Los datos de las personas fallecidas se obtuvieron mediante un "proxy"6 para reconstruir las condiciones de salud, económicas y de apoyo familiar durante el UAV. La obtención de información mediante proxy ha probado ser confiable, especialmente cuando se trata de medidas objetivas como estado de salud, limitaciones funcionales y características sociodemográficas (Neumann, Araki y Guterman, 2000). Otra particularidad de las respuestas mediante proxy es que las respuestas están condicionadas por la relación que haya tenido con la persona fallecida. Sin embargo, es la mejor fuente para indagar sobre las condiciones de salud, la presencia de incapacidades y los gastos incurridos. En las erogaciones se incluyen costos por visitas a clínicas y hospitales, medicamentos utilizados y consultas médicas realizadas. Se agregan datos de las personas que apoyaron económicamente. También se abordan preguntas sobre el lugar de muerte, la causa la muerte, y en caso de haber fallecido por alguna enfermedad se pregunta la causa principal. Se procuró que las preguntas fueran comparables con la información que el individuo proporcionó en la ronda anterior cuando estaba vivo, para comparar las características del individuo antes y durante su UAV. No se preguntó por limitaciones funcionales específicas, pero si se pregunta si hubo alguna dificultad para realizar alguna de las actividades básicas e instrumentales de la vida diaria.

Es importante señalar que los procesos estadísticos y las estimaciones resultantes se han elaborado con los datos directos de la muestra. No se ha aplicado factor alguno de expansión estadística, considerando que el grupo en estudio es la población total de los que han fallecido. Las variables examinadas son las sociodemográficas, condiciones de salud, el estado de funcionalidad y el gasto en el UAV. ENASEM tiene registrados un total de 4,496 fallecidos entre (2001-2015), pero se excluyeron debido a falta de información completa para analizar los gastos en salud. Así esta muestra son aquellos $\mathrm{n}=2,865$ individuos de la línea basal que en 2001 tenían 50 años o más y que fallecieron entre 2001 y 2015 y que contaba con información completa sobre el tipo de gasto en salud. La Tabla 2 distribuye el número de decesos entre rondas y por sexos. Se observa que la mayoría de las muertes se registra en la ronda 2012, explicable porque fallecieron en el período más amplio entre rondas, de nueve años, de 2003 a 2012.

\footnotetext{
${ }^{6}$ Se procuró que fuera el familiar que más se encargó de sus cuidados durante el UAV.
} 
Tabla 2: Tamaño y distribución por sexo de la muestra

\begin{tabular}{lrrrrrr}
\hline \multirow{2}{*}{ Ronda de UAV } & \multicolumn{2}{c}{ Hombres } & \multicolumn{3}{c}{ Mujeres } & \multicolumn{2}{c}{ Total } \\
& $\mathrm{n}$ & $\%$ & $\mathrm{n}$ & $\%$ & $\mathrm{n}$ & $\% *$ \\
\hline 2003 & 117 & 42.5 & 158 & 57.5 & 275 & 9.6 \\
2012 & 799 & 43.1 & 1,054 & 56.9 & 1,853 & 64.7 \\
2015 & 283 & 38.4 & 454 & 61.6 & 737 & 25.7 \\
Total & 1,199 & 41.8 & 1,666 & 58.2 & 2,865 & 100.0 \\
\hline
\end{tabular}

Fuente: elaboración propia con base en ENASEM 2001-2015.

Se ha comentado ya sobre la mayor longevidad en las mujeres, pero en peores condiciones de salud y funcionalidad que los hombres, por lo que se espera que las necesidades de atención médica y de cuidados sean distintas y más demandantes en las mujeres. Así una cuestión es cómo se afecta el UAV en razón de género, consideración siempre presente en este análisis.

En el cuestionario ENASEM de fallecidos ${ }^{7}$ la principal variable dependiente es el gasto en salud durante el UA. Se utilizan cuatro opciones de respuesta: sin gasto, gastos entre tres mil pesos y menos de seis mil, más de seis mil pesos, pero menos de 24 mil, y más de 24 mil pesos. Para al análisis se reclasificó en tres tipos de gasto según se muestra en la Tabla 3: sin gasto, bajo-medio (menos de 24 mil pesos MX) y alto (mayor a 24 mil pesos). De esta manera la interpretación no es sobre un montó concreto, sino de un tipo de gasto en el periodo de la ronda. Como se presenta en la Tabla 3, la mitad declararon no haber incurrido en gastos por salud, mientras que solamente 21.8 por ciento informó un gasto elevado.

Tabla 3: Tipo de gasto en salud durante el último año de vida, por sexo

\begin{tabular}{lrrrr}
\hline \multicolumn{1}{c}{ Ronda de UAV } & Hombres & \multicolumn{3}{c}{ Mujeres } \\
& $\mathrm{n}$ & $\%$ & $\mathrm{n}$ & $\%$ \\
\hline Sin gasto & 671 & 56.0 & 739 & 44.4 \\
Bajo-medio & 306 & 25.5 & 518 & 31.1 \\
Alto & 222 & 18.5 & 409 & 24.6 \\
Total & 1,199 & 100.0 & 1,666 & 100.0 \\
\hline
\end{tabular}

Fuente: elaboración propia con base en ENASEM 2001-2015.

${ }^{7}$ La pregunta se formula de la siguiente manera: "Nos interesa tener una idea muy aproximada del costo total de las hospitalizaciones, visitas a médicos, procedimientos, cirugías, medicinas, servicios especiales como contratar a alguien para cuidarlo (a), y demás gastos médicos que se hicieron por (Nombre) en el último año de vida, ¿diría usted que fueron...?”. 
Con los determinantes del tipo de gasto por sexo, se realizaron regresiones logísticas multinomiales, para hombres y mujeres, donde la variable dependiente puede ser binaria o de más categorías (Long, Freese; 2006). ${ }^{8}$ En estos modelos se utiliza como referencia la categoría "sin gasto". Las variables de control se dividen en sociodemográficas, de estado de salud y acceso a servicios de salud. En la sección de anexos, el Cuadro A.1 presenta la operacionalización de las variables. En las variables sociodemográficas se consideraron edad a la muerte, situación conyugal, escolaridad, lugar de muerte y localidad de residencia. Para el estado de salud las variables fueron: número de enfermedades durante el UAV, limitación en actividades básicas e instrumentales de la vida diaria, y causa de muerte (enfermedad, accidente o violencia). Se analizaron a su vez el número de enfermedades declaradas una ronda antes, y la enfermedad considerada causa primaria de muerte. Las variables de acceso a servicio de salud fueron el número de noches de hospital durante el UAV y el tipo de institución a la que se tiene acceso a servicios de salud.

\section{RESUltados}

Según aparecen en la encuesta, la Tabla 4 presenta características sociodemográficas, condiciones de salud y acceso a servicios de salud entre hombres y mujeres durante el UAV. En busca de diferencias entre sexos, estas cifras indican que la edad promedio a la muerte es 1.4 años mayor en hombres en comparación con las mujeres. Los porcentajes en edades 50 a 69 años son mayores entre mujeres, mientras que en hombres son mayores en 70 o más. Las principales diferencias son en situación de unión, escolaridad y localidad de residencia. 68.3 por ciento de las mujeres llegaron sin unión al UAV mientras que 49 por ciento de los hombres no estaban en unión. La escolaridad siempre se muestra mayor en hombres, incluyendo que en niveles de siete años de escolaridad o más es 3.3 por ciento superior. Respecto al lugar de residencia, 67.1 por ciento de las mujeres vivía en un área urbana, mientras que en los hombres fue 59.7 por ciento. Tanto hombres como mujeres en su mayoría fallecieron en sus casas 51.5 por ciento para ambos, solamente 47.1 por ciento de las mujeres fallecieron en un hospital o institución de cuidados, mientras que en los hombres fue 44.3 por ciento.

Es notorio que las mujeres tuvieron peores condiciones de salud. La proporción de mujeres con al menos una enfermedad crónica fue de 71.3 por ciento, 4.5 por ciento más que los hombres.

\footnotetext{
8 Se utilizó el software STATA 15 y RStudio para la visualización de los resultados.
} 
Determinantes de gastos en salud durante el último año de vida de la población ... / J.D. ZAZUETA y R. HAM

Tabla 4: Características de la encuesta en el UAV por sexos, con distribuciones porcentuales por columnas

\begin{tabular}{|c|c|c|c|}
\hline \multirow{2}{*}{ Variables } & \multicolumn{2}{|c|}{ Estadísticas descriptivas } & \multirow{2}{*}{$\begin{array}{l}\text { Diferencias } \\
\quad(\mathrm{H}-\mathrm{M})\end{array}$} \\
\hline & Hombres & Mujeres & \\
\hline \multicolumn{4}{|l|}{ Sociodemográficas } \\
\hline Muestra & 41.8 & 58.2 & \\
\hline Edad (media) & 78.6 & 77.2 & \\
\hline Edad Categoría (\%) & & & $* * *$ \\
\hline 50 a 59 & 2.9 & 5.1 & \\
\hline 60 a 69 & 16.7 & 21.9 & \\
\hline 70 a 79 & 31.9 & 27.7 & \\
\hline 80 o más & 48.5 & 45.3 & \\
\hline Unión (\%) & & & $* * *$ \\
\hline En unión & 50.8 & 31.7 & \\
\hline No & 49.2 & 68.3 & \\
\hline Escolaridad (\%) & & & $* * *$ \\
\hline 0 años & 32.5 & 36.2 & \\
\hline 1 a 6 & 53.1 & 52.6 & \\
\hline 7 o más & 14.4 & 11.1 & \\
\hline Localidad (\%) & & & $* * *$ \\
\hline Rural & 40.3 & 32.9 & \\
\hline Urbana & 59.7 & 67.1 & \\
\hline Lugar de muerte $(\%)$ & & & $* * *$ \\
\hline Casa & 51.5 & 51.4 & \\
\hline Hospital/Institución & 44.3 & 47.1 & \\
\hline Otro & 4.2 & 1.5 & \\
\hline \multicolumn{4}{|l|}{ Condiciones de salud } \\
\hline \multicolumn{4}{|l|}{ Número de enfermedades UAV (\%) } \\
\hline 0 & 33.2 & 28.7 & \\
\hline 1 & 42.1 & 46.5 & \\
\hline 2 & 20.1 & 20.1 & \\
\hline 3 o más & 4.6 & 4.8 & \\
\hline Limitación en $A B V D$ durante $U A V$ & & & $* * *$ \\
\hline No & 45.5 & 34.0 & \\
\hline Sí & 54.5 & 66.0 & \\
\hline
\end{tabular}


Tabla 4: Continuación

\begin{tabular}{|c|c|c|c|}
\hline & \multicolumn{2}{|c|}{ Estadísticas descriptivas } & \multirow{2}{*}{$\begin{array}{l}\text { Diferencias } \\
\quad(\mathrm{H}-\mathrm{M})\end{array}$} \\
\hline & Hombres & Mujeres & \\
\hline Limitación en AIVD durante UAV & & & $* * *$ \\
\hline No & 38.5 & 28.5 & \\
\hline Sí & 61.5 & 71.5 & \\
\hline Causa de muerte (\%) & & & n.s \\
\hline Enfermedad & 84 & 86 & \\
\hline Accidente/violencia u otra & 16 & 14 & \\
\hline Enfermedad de muerte (\%) & & & $* * *$ \\
\hline Cáncer & 15.6 & 14.86 & \\
\hline Diabetes & 10.43 & 20.37 & \\
\hline Embolia Cerebral & 5.79 & 5.44 & \\
\hline Corazón & 20.94 & 18.53 & \\
\hline Infección & 1.25 & 1.39 & \\
\hline Otra & 45.99 & 39.41 & \\
\hline \multicolumn{4}{|l|}{ Servicios de Salud } \\
\hline Número de visitas hospital UAV (\%) & & & $*$ \\
\hline 0 & 33.5 & 28.8 & \\
\hline 1 a 6 & 34.5 & 32.9 & \\
\hline 7 a 31 & 26.8 & 31.8 & \\
\hline 32 o más & 5.2 & 6.5 & \\
\hline Afiliación a servicios de salud (\%) & & & n.s \\
\hline Sin acceso & 14.7 & 14.9 & \\
\hline Seguro Popular & 14.7 & 15.2 & \\
\hline Servicios Estatales & 67.4 & 65.8 & \\
\hline Servicios Privados & 3.2 & 4.2 & \\
\hline \multicolumn{4}{|l|}{ Gastos en salud } \\
\hline Sin gasto & 56.0 & 44.4 & \\
\hline Bajo-Medio & 25.5 & 31.1 & \\
\hline Alto & 18.5 & 24.6 & \\
\hline
\end{tabular}

n.s. $=$ no significativa.

$* p<=0.05 ; * * p<=0.005 ; * * * p<=0.001$

Fuente: elaboración propia con base en ENASEM 2001 y 2015. 
Las necesidades de cuidado en las Actividades Básicas de la Vida Diaria (ABVD) y en las Actividades Instrumentales de la Vida Diaria (AIVD) fue 11.5 por ciento y 10.1 por ciento superior en mujeres. Otra diferencia es que la principal causa de muerte en las mujeres fue diabetes, y para los hombres son las enfermedades isquémicas del corazón.

La información sobre acceso a servicios de salud muestra que 71.2 por ciento de las mujeres pasó al menos una noche de hospital, mientras que fue 66.5 por ciento en hombres. La afiliación a servicios de salud no tiene diferencias mayores entre sexos. La mayoría cuentan con servicios estatales (IMSS, ISSSTE, Seguro Popular o Pemex). Los gastos en salud fueron mayores en las mujeres.

El Cuadro A.2 del anexo presenta la asociación entre gastos de salud y los diferentes determinantes analizados, junto con los resultados de modelos de regresión logística multinomial que analizan el efecto de los diferentes determinantes sobre el gasto en salud, para hombres y mujeres por separado. Es información que se vierte en las siguientes tres figuras.

La edad ejerce un efecto diferente entre hombres y mujeres en el riesgo de experimentar un gasto bajo-medio o alto en salud. En ambos tipos de gasto, las mujeres de 80 años o más son más propensas a gastos en comparación con los hombres, especialmente cuando el gasto es alto. Los efectos de escolaridad, localidad, y lugar de muertes siguen el mismo patrón para hombres y mujeres, con excepción de la categoría de "otro en lugar" de muerte para las mujeres, aunque su efecto no es estadísticamente significativo. En términos de unión se observa diferencia pues los hombres unidos son más propensos a reportar gasto alto en comparación con los que no están en unión, mientras que en las mujeres no hubo diferencia (Figura 2).

Las cifras apuntan a que las mujeres llegan en condiciones de salud más deterioradas en comparación con los hombres. Sin embargo, los efectos de las condiciones de salud sobre las probabilidades de experimentar algún tipo de gasto son similares entre hombres y mujeres como se ve en la Figura 3. La única diferencia es que en los hombres el efecto de fallecer de alguna enfermedad y no de muerte accidental o violenta ocasionan un gasto bajo-medio, mientras que en las mujeres el efecto es inverso. También el contar con tres enfermedades o más en el caso de los hombres incrementa 2.5 veces el riesgo de gasto alto, mientras que en las mujeres el efecto es poco notorio, de 1.3. Para ambos, el efecto de contar con alguna limitación en las AIVD incrementa el riesgo de experimentar ambos tipos de gasto, especialmente para hombres ya que es 3.9 veces mayor el gasto alto. Este factor es 1.7 en las mujeres. 


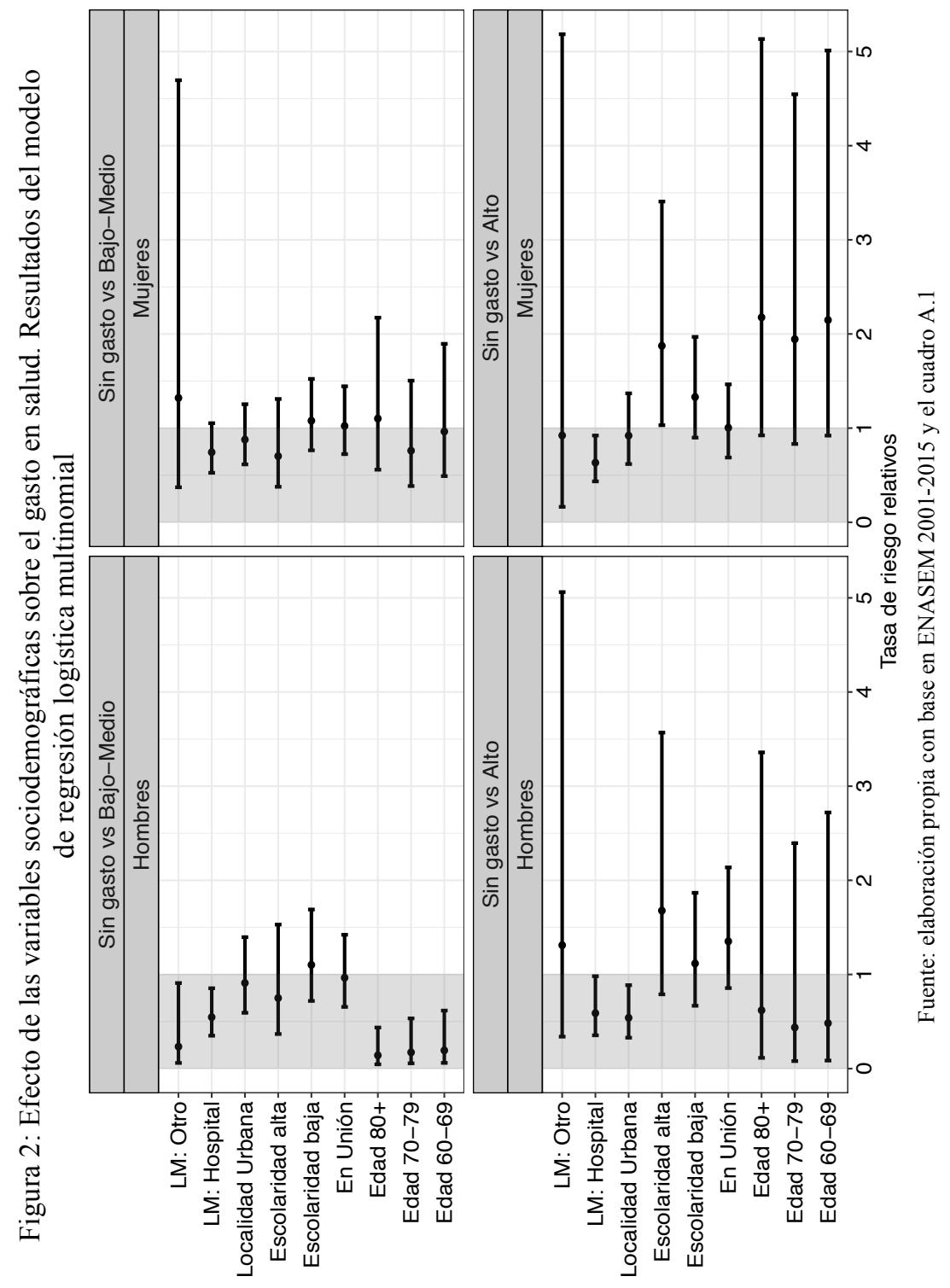


Determinantes de gastos en salud durante el último año de vida de la población ... / J.D. ZAZUETA y R. HAM

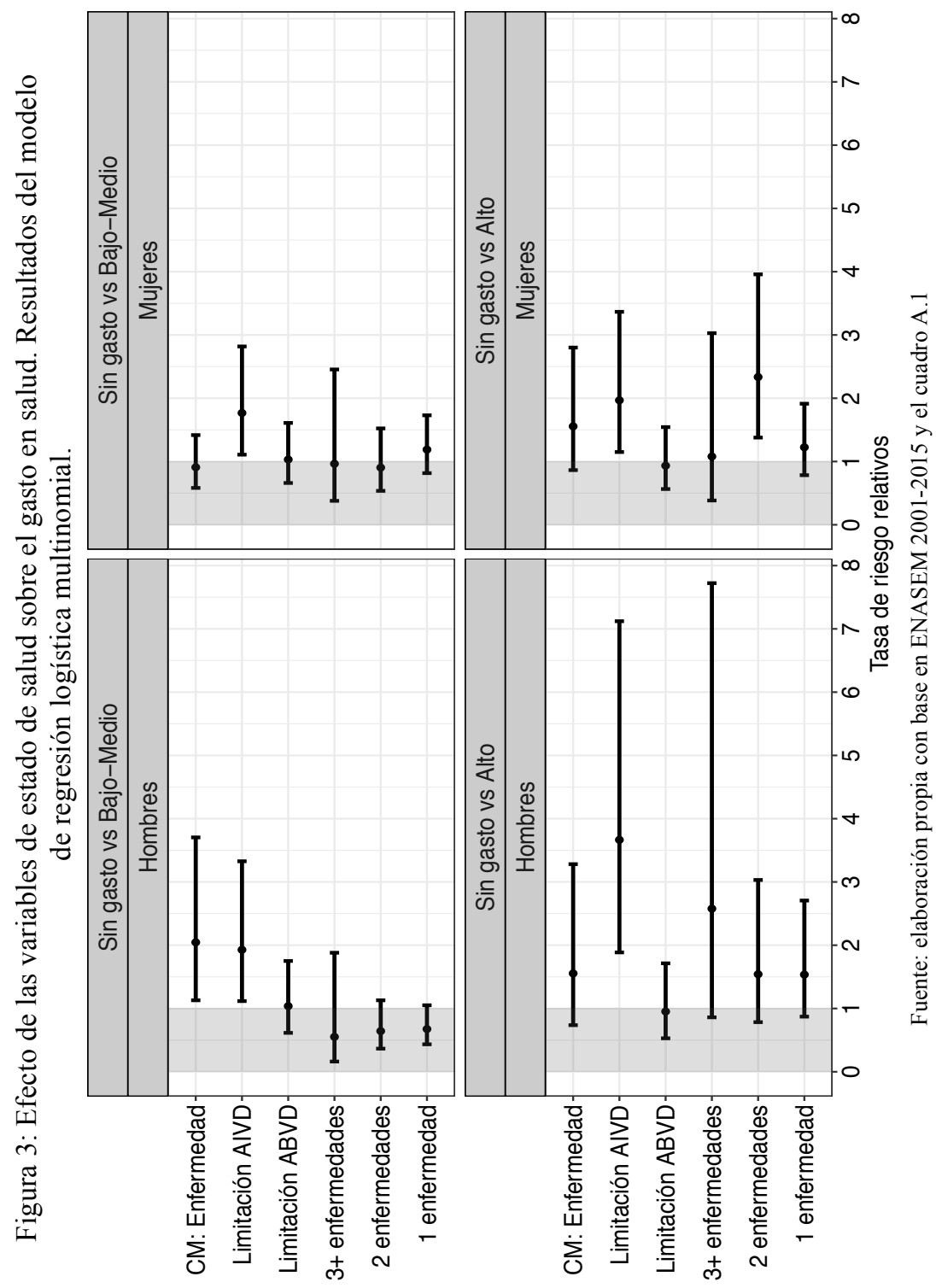


Tanto para hombres y mujeres el principal determinante del gasto alto en salud fueron el número de noches de hospital. En la Figura 4, se presentan los efectos de las variables de número de noches de hospital y de acceso a servicios de salud. Tanto para hombres como mujeres el pasar al menos una noche en el hospital incrementa las probabilidades de gasto en el UAV. De la misma manera, contar con acceso a servicios estatales de salud reduce el riesgo de tener que experimentar algún tipo de gasto.

Si bien el efecto de noches de hospital sobre el gasto en salud durante el UAV es similar entre hombres y mujeres, la Figura 5 muestra que las probabilidades de experimentar al menos una noche de hospital no siguen el mismo patrón por edad y número de enfermedades entre ambos sexos. Independientemente del número de enfermedades, para las mujeres el riesgo de pasar al menos una noche de hospital decrece con la edad, y el grupo de edad con mayor probabilidad es de 50 a 59 años independientemente del número de enfermedades crónicas reportadas. En cambio, para los hombres la probabilidad de pasar al menos una noche en el hospital incrementa con la edad y alcanza su punto máximo en el grupo de edad de 70 a 79 años y después algo decrece en el grupo de 80 años o más.

\section{CONClusiones y PROPUESTAS}

El envejecimiento demográfico espera más personas en edades avanzadas y con mayor longevidad. Así, su presencia en hogares y redes familiares será mayor (García, 2014) incrementando el riesgo de gastos por cuenta propia por motivos de salud. El mayor efecto sobre los determinantes del gasto señala como factores principales al deterioro del estado de salud y las noches de hospital (Ceballos, 2019; Granados y Nava, 2019). Los resultados de esta investigación son consistentes con estas investigaciones previas, sin embargo, se agrega que los mayores riegos y gastos son durante el UAV con las noches de hospital como el principal factor del gasto. También se documentó la relación entre el número de enfermedades y las noches de hospital, señalando que conforme la edad es mayor y el estado de salud se deteriora las probabilidades de pasar al menos una noche de hospital se incrementan, aunque en patrones por edad que varían entre hombres y mujeres. Una limitación de los hallazgos es que no logran establecer la relación causal entre el deterioro de salud y las noches de hospital y por tanto de la mayor probabilidad de gasto, aunque que se documentó la relación entre las variables. 
Determinantes de gastos en salud durante el último año de vida de la población ... / J.D. ZAZUETA y R. HAM
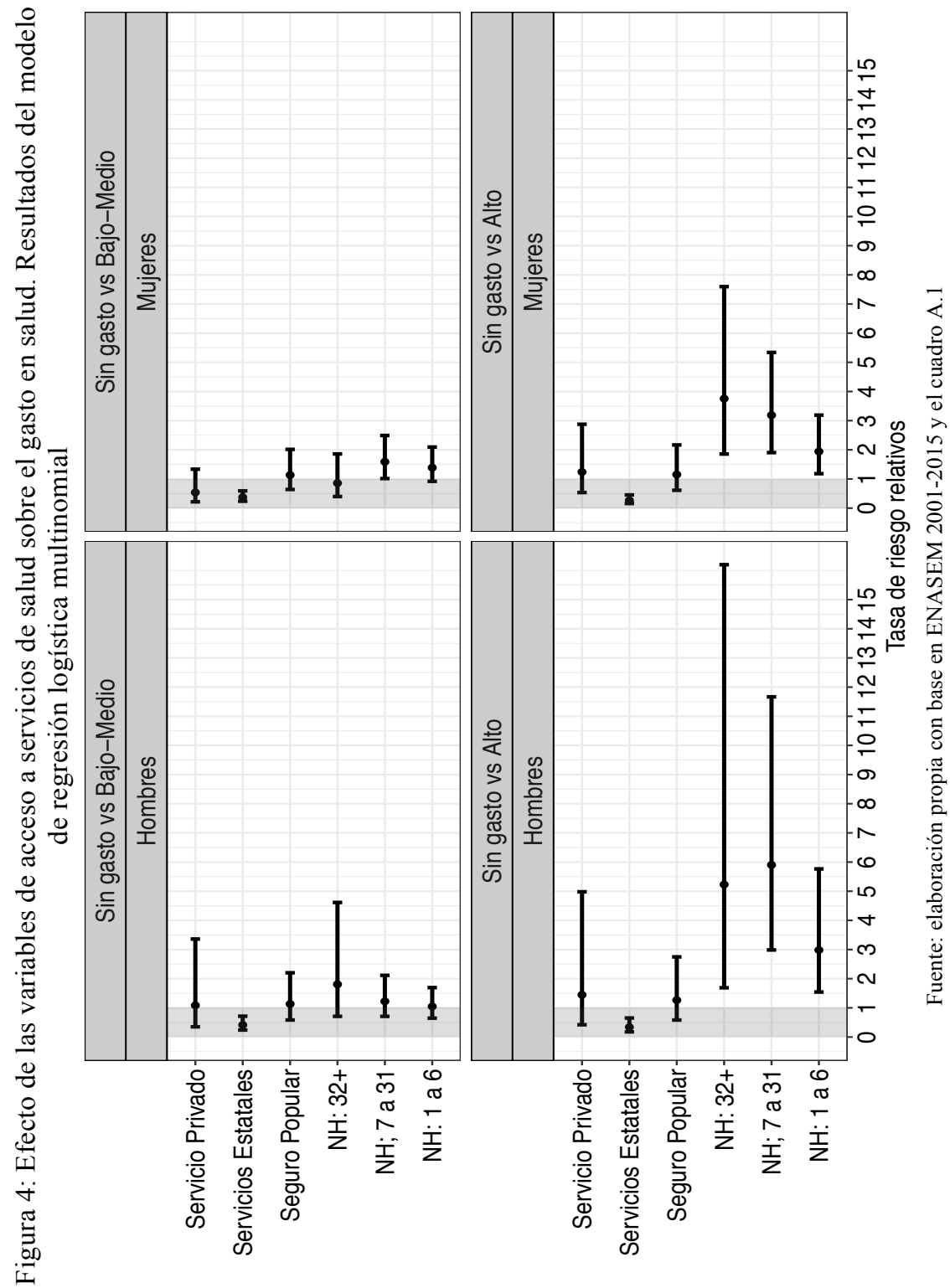


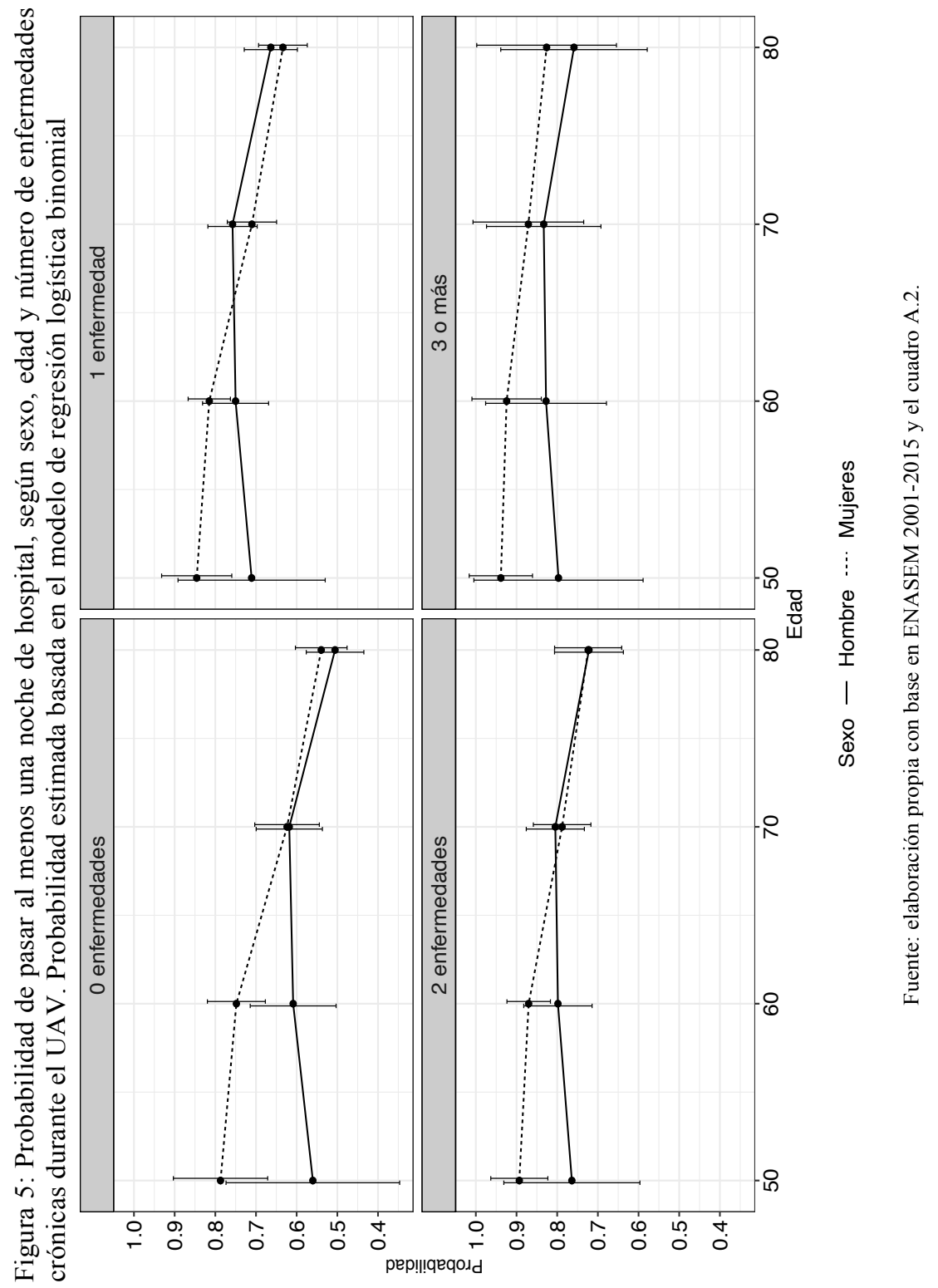


Un elemento central en el análisis de los determinantes del gasto es la condición de afiliación a servicios de atención médica. Granados y Nava (2019) encontraron, usando la Encuesta Nacional de Ingresos y Gastos en los Hogares para los años de 2008 a 2014, que el acceso a servicios de salud reduce el riesgo de gastos catastróficos. Sus resultados son similares a los de Knaul et al. (2018), quienes indican que el Seguro Popular tenía un efecto positivo en la reducción de riesgos de gastos al ofrecer protección financiera. Sin embargo, en este estudio la afiliación a servicios estatales como IMSS, ISSSTE y Pemex, fueron los únicos que reducen el riesgo de tener un gasto alto. De hecho, la variable del Seguro Popular indica propensión a gasto mayor, aunque no es estadísticamente significativa.

Gonzáles et al. (2018) usando el ENASEM para las rondas 2003 a 2012 señalan, sobre el UAV, que uno de los determinantes principales en el número de noches de hospital son las limitaciones funcionales, algunas causas de muerte como cáncer y la necesidad de cuidados. Sin embargo, para este artículo no se encontró efecto de las causas de muerte ${ }^{9}$ sobre el gasto, pero si por parte de las AIVD y por el hecho de fallecer de alguna enfermedad y no por muerte accidental o violenta. En la misma línea, los resultados son similares a los de Salinas et al. (2019), señalando que las personas que cuentan con acceso a servicios de salud tienen menos gasto. La diferencia de este análisis con respecto a los anteriores es que se optó por analizar al gasto mediante categorías. La razón principal fue analizar las características de las personas que no tuvieron gastos y señalar la magnitud de la influencia de los determinantes del gasto en comparación con los que sí tuvieron gastos. Asimismo, se separó el análisis entre hombres y mujeres, partiendo de la premisa de que las condiciones de salud en el UAV son diferentes.

Dentro de las investigaciones que se han realizado en Europa usando la Encuesta de Salud, Envejecimiento y Jubilación en Europa (SHARE), encuesta similar a ENASEM, Orlovic et al. (2018) se encontró que el principal determinante del gasto por cuenta propia se asocia con el lugar de la muerte y las políticas de cuidados paliativos, encontrando una relación entre el PIB destinado en salud y el porcentaje de muertes en hospital. En ENASEM los resultados sobre la influencia del lugar de la muerte no son del todo claras, pues al parecer, aunque se hayan pasado noches de hospital

\footnotetext{
${ }^{9}$ En este trabajo se también se analizaron las enfermedades de causa de muerte que utilizan Gonzáles et al. (2018) y Salinas et al. (2019), en los análisis de regresión. Sin embargo, se optó solo por presentar en el análisis si la causa de muerte fue una enfermedad o muerte accidental o violenta. Ya que ni ninguna de las causas de muerte tuvo una mayor influencia en el tipo de gasto. Los resultados de dichos modelos se pueden solicitar al autor principal.
} 
durante el UAV la propensión es a fallecer en casa, quizá porque en México aún no se consolidan políticas de cuidados paliativos a largo plazo.

En esta investigación una hipótesis fue que el acceso a servicios de salud es la determinante principal del gasto. Los resultados sugieren que influye, pero es más ilustrativo el número de noches de hospital como principal determinante. Factores asociados con el deterioro de salud manifestado por limitaciones funcionales o el número de enfermedades crónicas también se relacionan con el gasto. Sin embargo, las condiciones de estado de salud incrementan las probabilidades de noches de hospital, lo que indica que el efecto que tienen sobre los gastos es indirecto. Finalmente, los resultados son consistentes con investigaciones previas, tanto aquellas que abordan los determinantes del gasto para toda la población, como para las que se concentran en los adultos mayores durante el UAV. Otro hallazgo importante es que las mujeres son más propensas a tener gastos durante el UAV debido a que las mujeres llegan con peor estado de salud y requieren mayor atención hospitalaria.

Sin bien este trabajo aporta a la literatura sobre la dinámica del gasto durante el UAV, es importante señalar limitaciones. Por ejemplo, no se documenta el efecto que tienen estos gastos con el riesgo de gastos catastróficos, principalmente debido a las limitaciones para vincular los ingresos individuales y de familiares cuando no se vive en el mismo hogar. También se tiene evidencia de que el ahorro es una fuente de ingreso de los hogares para hacer frente a este tipo de situaciones (Ceballos, 2019). Desafortunadamente ENASEM no cuenta con esa información. Una última limitación es que aquí sólo se analiza el gasto realizado por individuos. Una imagen más clara del impacto económico del UAV debería contar con información de registros administrativos de programas e instituciones de apoyo (Hogan et al., 2001). ${ }^{10}$

ENASEM es la única fuente de información para conocer el UAV de los adultos mayores, con la fortaleza de ser un estudio representativo a nivel nacional para la población de 50 años o más. Si bien se mencionaron algunas de sus limitaciones, los resultados que se obtuvieron permiten una imagen sobre cómo hombres y mujeres experimentan el UAV. El UAV en México, como señala González y otros (2018) es muy heterogéneo. Una línea futura de investigación de este tema podría aprovechar los datos longitudinales de la ENASEM para analizar trayectorias de deterioro de salud

${ }^{10}$ Esto permitirá comparar los resultados de programas, como el Medicare en los Estados Unidos sobre los gastos en salud durante el último año de vida, ya que en Estados Unidos se ha documentado que los beneficiarios de dicho programa utilizan una cuarta parte del dinero asignado por el Medicare en el último año de vida. 
y acceso a servicios de salud. Analizar temas específicos y de manera longitudinal llevaría a planteamientos, análisis y conclusiones más claras que además tengan sintonía con otros temas que así generen políticas públicas para mejorar las condiciones de salud y reducir las desigualdades en el acceso a servicios de salud como una estrategia para hacer frente a los retos económicos y sociales del envejecimiento demográfico.

\section{Agradecimientos}

Agradecemos a Hiram Beltrán Sánchez, Silvia Mejía y Cesar González, por sus comentarios en una versión previa a este documento. También agradecemos al equipo de ENASEM (Estudio Nacional de Salud y Envejecimiento en México). Este estudio es financiado parcialmente por el National Institutes of Health/National Institute on Aging (NIH R01AGO18016) en Estados Unidos y el Instituto Nacional de Estadística y Geografía (INEGI) en México. Los archivos de datos y la documentación son uso público y están disponibles en www.ENASEM.org.

\section{REFERENCIAS BIBLIOGRÁFICAS}

Aldridge, M. y Kelly, S., 2015, "The myth regarding the high cost of end-of-life care", in American Journal of Public Health, vol. 105, núm. 12.

Barba, C., 2013, "Inseguridad y protección social en los países desarrollos y en América Latina", en Revista Mexicana de Sociología, vol. 71, núm. 1.

Barba, C., 2015, "Universalist reform of the Health Systems in Mexico and Chile. A change of time or historical inertia?", in Journal of Public Governance and Policy: Latin America Review, vol. 1, núm. 2.

Ceballos, O. E., 2019, "Perfiles económicos y comportamiento del gasto en salud de los hogares con personas adultas mayores", en Estudios Demográficos y Urbanos, vol. 34, núm. 3 .

Diaz, C. y otros, 2016, "The progression of Disability among Older Adults in Mexico", in Disability and Rehabilitation, vol. 38, núm. 2.

Diaz, C. y Wong, R., 2016, "Trajectories of Limitations in Activities of Daily Living among Older Adults in Mexico, 2001-2012", in Disability and Health Journal, vol. 9.

García, V. M., 2014, Proyecciones y políticas de población en México. México. El Colegio de México.

González, C. y otros, 2018, "Envejecimiento y mortalidad: condiciones de salud, económicas y familiares en el último año de vida de las personas mayores en México", en Papeles de Población, vol. 24, núm. 97. 
Granados, M. y Nava, I., 2019, “Gastos catastróficos por motivos de salud y hogares con personas mayores en México”, en Papeles de Población, vol. 25, núm. 99.

INEGI, 2015, Encuesta Intercensal 2015, Instituto Nacional de Estadística y Geografía, disponible en https://www.inegi.org.mx/programas/intercensal/2015/default,html\#Tabulados

INEGI, 2001, Indicadores Sociodemográficos de México (1930-2000), Instituto Nacional de Estadística y Geografía, México. Disponible en http://internet.contenidos.inegi.org.mx/contenidos/Productos/prod_serv/contenidos/espanol/bvinegi/ productos/historicos/2104/702825493875/702825493875_1.pdf

INEGI, 2019, Presentación de resultados de la Encuesta Nacional de la Dinámica Demográfica en México, ENADID 2018, disponible en https://www.inegi.org.mx/ contenidos/programas/enadid/2018/doc/resultados_enadid18,pdf

Ham, R., 2003, El envejecimiento en México: el siguiente reto de la transición demográfica, Miguel Ángel Porrúa y Colegio de la Frontera Norte.

Hogan, C. y otros, 2001, "Medicare Beneficiaries Costs of Care in the Last Year of Life", in Health Affairs, vol. 20, núm. 4.

Knaul F. y otros, 2001, "Househould catastrophic health expenditure: A comparative analysis of twelve Latin American and Caribbean Countries", en Salud Pública de México, vol. 53.

Knaul F. y otros, 2018, "Efecto del Seguro Popular de Salud sobre los gastos catastróficos y empobrecedores en México, 2004-2012”, en Salud Pública de México, vol, 60 .

Long, S. y Freese, J., 2006, Regression Models for Categorical Dependent Variable Using Stata, Texas: Stata Press.

Luna, M., 2015, “Cuando el aseguramiento en salud no basta, ¿quiénes gastan más en servicios y atención a la salud?”, en Coyuntura Demográfica, núm. 7.

Moreno, J. y Flamand, L., 2015, “Towards health-care equality? The perfomance of seguro popular in Mexico (2003-2013)", in Journal of Public Governance and Policy: Latin America Review, vol. 1, núm. 2.

Neumann, P., Araki, S. y Gutterman, E., 2000, "The use of proxy respondents in studies of older adults: lessons, challenges, and opportunities", in Journal of the American Geriatrics Society, vol. 48, No 12.

OMS, 2005, Designing Health Financing System to Reduce Catastrophic Health Expenditures, World Health Organization, disponible en https://apps.who.int/iris/ bitstream/handle/10665/70005/WHO_EIP_HSF_PB_05.02_eng.pdf;jsession$\mathrm{id}=\mathrm{D} 838386 \mathrm{C} 6 \mathrm{DDC} 4 \mathrm{~B} 2076 \mathrm{~B} 36 \mathrm{CC} 3 \overline{8} 117 \overline{0} 67 \mathrm{~B}$ ? sequence $=\overline{1}$

Orlovic, M., Marti, J. y Mossialos, E., 2017, “Analysis of end-of-life care, out-ofpocket spending, and place of death in 16 European Countries and Israel", Health Affairs, vol. 36, núm. 7.

Orozco, K., Wong, R. y Michaels, A., 2018, "Atrición en encuestas de panel en México: La Encuesta Nacional sobre Salud y Envejecimiento en México 
(ENASEM)", en Realidad, Datos y Espacio, Revista Internacional de Estadística y Geografía, vol. 9, núm. 1.

Parker, W., Saenz, J. y Wong, R., 2017, "Health insurance and the aging: Evidences from the Seguro Popular program in México", in Demography, vol. 55.

Penders, Y. y otros, 2017, "Differences in out-of-pocket costs of healthcare in the last year of life of older people in 13 European countries", in Palliative Medicine, vol. 31 núm. 1.

Salinas, G. y otros, 2019, "Gastos de bolsillo en salud durante el último año de vida de adultos mayores mexicanos: análisis de ENASEM", en Salud Pública de México, vol. 61.

Valencia, E., Foust, D. y Tetreault, D., 2012, Social protections systems in Latin America and the Caribean, México, Santiago de Chile. Publicación de las Naciones Unidas.

Verbrudgge, L., 2016, "Disability experiences and measurement", in Journal of Aging and Health, vol. 28, núm. 7.

Zazueta, J. D., 2018, Cambios en los gastos en salud y tipo de gasto durante el último año de vida de la población de 60 años y más en México, Tesis de Maestría en Estudios de Población, El Colegio de la Frontera Norte.

\section{RESUMEN CURRICULAR DE LOS AUTORES}

Jesús Daniel Zazueta Borboa

Estudiante de Doctorado en Demografía en el Netherlands Interdisciplinary Demographic Institute, en La Haya, Holanda y estudiante del programa Poblacion, Salud y Ciencia del Datos del Max Planck Institute for Demographic Research, en Rostock Alemania. Egresado de la Licenciatura en Sociología por la Universidad de Guadalaja y Maestro en Estudio de Población por El Colegio de la Frontera Norte, cursó el programa de European Doctoral School of Demography en el Centro de Estudios Demográficos de la Universidad Autónoma de Barcelona y el Max Planck Institute for Demographic Research. Su investigación gira entorno a la mortalidad, envejecimiento demográfico y las desigualdades socioeconómicas en salud y en la esperanza de vida en América Latina y Europa. Previo al doctorado, trabajó como asistente de investigación en la Universidad de Guadalajara y en el área de información y estadística del Consejo de Evaluación de la Política Social del Gobierno de la Ciudad de México.

Dirección electrónica: Zazueta@nidi.nl

Registro ORCID: https://orcid.org/0000-0003-4132-1057 


\section{Roberto Ham-Chande}

Actuario de la Facultad de Ciencias, UNAM. Maestría en Estadística Matemática, Centro Interamericano de Enseñanza de Estadística, Santiago, Chile. Maestría en Ciencias, Department of Statistics and Probability, Michigan State University. Doctor en Demografía, Université de Paris Nanterre, con la máxima mención honorífica. Es profesor-investigador del Departamento de Estudios en Población en El Colegio de la Frontera Norte y profesor honorario de El Colegio de México. Ha sido actuario del IMSS y del ISSSTE. Ex-director del Centro de Estudios Demográficos y Desarrollo Urbano de El Colegio de México. Pertenece al Sistema Nacional de Investigadores desde 1985, actualmente en nivel 3. Premio Nacional de Demografía 2009.

Dirección electrónica: rham@colef.mx 


\section{Anexo}

Cuadro A.1: Operacionalización de las variables

\begin{tabular}{|c|c|c|c|}
\hline Determinantes & Dimensión & Variables & Valores \\
\hline \multirow{14}{*}{ Sociodemográficos } & \multirow{4}{*}{ Edad a la muerte } & $50-59$ & 0 \\
\hline & & 60 a 69 & 1 \\
\hline & & 70 a 79 & 2 \\
\hline & & 80 o más & 3 \\
\hline & \multirow{2}{*}{ Unión } & En unión & 0 \\
\hline & & No en unión & 1 \\
\hline & \multirow{3}{*}{ Escolaridad } & 0 & 0 \\
\hline & & 1 a 6 & 1 \\
\hline & & 7 o más & 2 \\
\hline & \multirow{3}{*}{ Lugar de muerte } & Casa & 0 \\
\hline & & Hospital & 1 \\
\hline & & Otro & 2 \\
\hline & \multirow{2}{*}{ Localidad } & Rural & 0 \\
\hline & & Urbana & 1 \\
\hline \multirow{10}{*}{ Estado de salud } & \multirow{4}{*}{$\begin{array}{l}\text { Número de enfermedades } \\
\text { en el UAV }\end{array}$} & 0 & 0 \\
\hline & & 1 & 1 \\
\hline & & 2 & 2 \\
\hline & & 3 o más & 3 \\
\hline & \multirow{2}{*}{ Causa muerte } & Enfermedad & 0 \\
\hline & & Accidente/violencia u otra & 1 \\
\hline & \multirow{4}{*}{ Con limitaciones } & Sin limitación ABVD & 0 \\
\hline & & Con limitación ABVD & 1 \\
\hline & & Sin limitación AIVD & 0 \\
\hline & & Con limitación AIVD & 1 \\
\hline \multirow{8}{*}{ Servicios de salud } & \multirow{4}{*}{ Visitas al hospital UAV } & 0 & 0 \\
\hline & & 1 a 6 & 1 \\
\hline & & 7 a 31 & 2 \\
\hline & & $32+$ & 3 \\
\hline & \multirow{4}{*}{$\begin{array}{l}\text { Acceso a servicios de } \\
\text { salud }\end{array}$} & Sin acceso & 0 \\
\hline & & Seguro Popular & 1 \\
\hline & & Estatales & 2 \\
\hline & & Privada & 3 \\
\hline
\end{tabular}

Fuente: elaboración propia con base en ENASEM 2001-2015. 
Cuadro A.2. Modelo de regresión logística múltinomial por sexo. Riesgos relativos y sus intervalos de confianza del $95 \%$ de los coeficientes en paréntesis.

\begin{tabular}{|l|c|c|c|c|}
\hline & \multicolumn{2}{|c|}{ Hombres } & \multicolumn{2}{c|}{ Mujeres } \\
\hline Variables & $\begin{array}{c}\text { Sin gasto vs } \\
\text { Bajo-medio }\end{array}$ & $\begin{array}{c}\text { Sin gasto } \\
\text { vs Alto }\end{array}$ & $\begin{array}{c}\text { Sin gasto vs } \\
\text { Bajo-medio }\end{array}$ & $\begin{array}{c}\text { Sin gasto vs } \\
\text { Alto }\end{array}$ \\
\hline Sociodemográficas \\
\hline Edad a la muerte (50 a 59 ref.) \\
\hline 60 a 69 & $0.147^{* *}$ & 0.66 & 1.23 & $3.49^{*}$ \\
\hline & $(0.04-0.53)$ & $(0.06-6.41)$ & $(0.53-2.81)$ & $(1.09-11.14)$ \\
\hline 70 a 79 & $0.14 * *$ & 0.54 & 1.04 & 2.97 \\
\hline & $(0.04-0.51)$ & $(0.05-5.16)$ & $(0.45-2.38)$ & $(0.93-9.46)$ \\
\hline 80 a 89 & $0.12^{* * *}$ & 0.77 & 1.58 & $3.59 *$ \\
\hline & $(0.03-0.42)$ & $(0.08-7.25)$ & $(0.69-3.59)$ & $(1.12-11.49)$ \\
\hline
\end{tabular}

Situación conyugal (En unión ref.)

\begin{tabular}{|l|c|c|c|c|}
\hline No en unión & 1.04 & 0.85 & 1 & 1.19 \\
\hline & $(0.68-1.58)$ & $(0.52-1.38)$ & $(0.69-1.45)$ & $(0.79-1.81)$ \\
\hline
\end{tabular}

Escolaridad (0 años ref.)

\begin{tabular}{|l|c|c|c|c|}
\hline 1 a 6 & 1.1 & 1.02 & 1.02 & 1.4 \\
\hline & $(0.68-1.74)$ & $(0.59-1.76)$ & $(0.71-1.46)$ & $(0.91-2.14)$ \\
\hline 7 o más & 0.94 & 1.54 & 0.51 & $1.91^{*}$ \\
\hline & $(0.44-1.98)$ & $(0.69-3.41)$ & $(0.24-1.04)$ & $(0.99-3.66)$ \\
\hline
\end{tabular}

Lugar de muerte (Casa ref.)

\begin{tabular}{|c|c|c|c|c|}
\hline Hospital & $0.54 *$ & $0.57 *$ & 0.78 & $0.60 *$ \\
\hline & $(0.34-0.88)$ & $(0.33-0.98)$ & $(0.53-1.12)$ & $(0.41-0.91)$ \\
\hline \multirow[t]{2}{*}{ Otro } & 0.26 & $1.13 *$ & 1.16 & 1 \\
\hline & $(0.06-1.02)$ & $(0.24-5.17)$ & $(0.28-4.71)$ & $(0.16-5.96)$ \\
\hline \multicolumn{5}{|c|}{ Localidad (Rural ref.) } \\
\hline \multirow[t]{2}{*}{ Urbana } & 0.71 & $0.50 *$ & 0.89 & 0.93 \\
\hline & $(0.44-1.11)$ & $(0.31-0.85)$ & $(0.61-1.29)$ & $(0.61-1.42)$ \\
\hline \multicolumn{5}{|c|}{ Estado de salud } \\
\hline \multicolumn{5}{|c|}{ Número de enfermedades en el UAV (0 ref.) } \\
\hline \multirow[t]{2}{*}{1} & $0.61^{*}$ & 1.54 & 1.14 & 1.32 \\
\hline & $(0.37-0.98)$ & $(0.84-2.81)$ & $(0.76-1.71)$ & $(0.81-2.15)$ \\
\hline \multirow[t]{2}{*}{2} & 0.72 & 1.66 & 0.91 & $2.74 * * *$ \\
\hline & $(0.39-1.31)$ & $(0.81-3.38)$ & $(0.51-1.59)$ & $(1.54-4.88)$ \\
\hline
\end{tabular}

Continúa 
Cuadro A.2. Continuación

\begin{tabular}{|l|c|c|c|c|}
\hline & \multicolumn{2}{|c|}{ Hombres } & \multicolumn{2}{c|}{ Mujeres } \\
\hline Variables & $\begin{array}{c}\text { Sin gasto vs } \\
\text { Bajo-medio }\end{array}$ & $\begin{array}{c}\text { Sin gasto } \\
\text { vs Alto }\end{array}$ & $\begin{array}{c}\text { Sin gasto vs } \\
\text { Bajo-medio }\end{array}$ & $\begin{array}{c}\text { Sin gasto vs } \\
\text { Alto }\end{array}$ \\
\hline 3 o más & 0.63 & 2.46 & 0.94 & 1.25 \\
\hline & $(0.17-2.21)$ & $(0.76-7.94)$ & $(0.34-2.52)$ & $(0.43-3.66)$ \\
\hline
\end{tabular}

\begin{tabular}{|l|c|c|c|c|}
\hline \multicolumn{6}{|l}{ Limitacionales funcionales (Sin limitación ref.) } \\
\hline ABVD & 1.01 & 0.98 & 0.9 & 0.98 \\
\hline & $(0.57-1.77)$ & $(0.53-1.81)$ & $(0.55-1.44)$ & $(0.56-1.71)$ \\
\hline AIVD & $2.08^{*}$ & $3.90^{* * *}$ & $1.80^{*}$ & 1.65 \\
\hline & $(1.15-3.74)$ & $(1.94-7.84)$ & $(1.09-2.97)$ & $(0.92-2.95)$ \\
\hline
\end{tabular}

Causa muerte (enfermedad ref.)

\begin{tabular}{|c|c|c|c|c|}
\hline $\begin{array}{l}\text { Accidente/violencia u } \\
\text { otra }\end{array}$ & $2.01^{*}$ & 1.9 & 0.93 & 1.46 \\
\hline & $(1.06-3.81)$ & $(0.84-4.26)$ & $(0.57-1.51)$ & $(0.77-2.73)$ \\
\hline \multicolumn{5}{|l|}{ Servicios de salud } \\
\hline \multicolumn{5}{|c|}{ Visitas al hospital UAV (0 ref.) } \\
\hline \multirow[t]{2}{*}{1 a 6} & 0.94 & $2.44 *$ & 1.24 & $1.87 *$ \\
\hline & $(0.56-1.57)$ & $(1.23-4.81)$ & $(0.79-1.92)$ & $(1.09-3.21)$ \\
\hline \multirow[t]{2}{*}{7 a 31} & 1.19 & $5.16^{* * *}$ & 1.6 & $3.25 * * *$ \\
\hline & $(0.65-2.14)$ & $(2.53-10.55)$ & $(0.99-2.57)$ & $(1.85-5.69)$ \\
\hline \multirow[t]{2}{*}{$32+$} & 1.27 & $4.51 *$ & 0.73 & $4.44 * * *$ \\
\hline & $(0.44-3.62)$ & $(1.41-14.38)$ & $(0.31-1.79)$ & $(2.06-9.58)$ \\
\hline
\end{tabular}

Acceso a servicios de salud (Sin acceso ref.)

\begin{tabular}{|l|c|c|c|c|}
\hline Seguro Popular & 1.29 & 1.29 & 1.16 & 1.31 \\
\hline & $(0.63-2.63)$ & $(0.57-2.91)$ & $(0.63-2.13)$ & $(0.66-2.58)$ \\
\hline Estatales & $0.44 *$ & $0.32 * * *$ & $0.36^{* * *}$ & $0.25^{* * *}$ \\
\hline & $(0.24-0.81)$ & $(0.16-0.65)$ & $(0.22-0.61)$ & $(0.14-0.45)$ \\
\hline Privados & 1.17 & 1.21 & 0.57 & 1.45 \\
\hline Constante & $(0.34-3.99)$ & $(0.32-4.56)$ & $(0.21-1.57)$ & $(0.56-3.71)$ \\
\hline & 3.07 & 0.1 & 0.64 & $0.05 * * *$ \\
\hline & $(0.71-13.17)$ & $(0.01-1.17)$ & $(0.23-1.78)$ & $(0.01-0.21)$ \\
\hline
\end{tabular}

$* \mathrm{P}<0.05 ; * * \mathrm{P}<0.005 ; * * * \mathrm{P}<0.001$

Fuente: elaboración propia con base en ENASEM 2001-2015. 
Cuadro A.3: Coeficientes del modelo de regresión logística binomial, sobre el pasar al menos una noche de hospital durante el UAV, intervalos de confianza del 95\% de los coeficientes en paréntesis. Hombres y Mujeres.

\begin{tabular}{|c|c|c|}
\hline Variables & Hombres & Mujeres \\
\hline \multicolumn{3}{|c|}{ Edad a la muerte (50-59 ref.) } \\
\hline \multirow[t]{2}{*}{60 a 69} & 1.88 & 0.95 \\
\hline & $(0.64-5.51)$ & $(0.41-2.17)$ \\
\hline \multirow[t]{2}{*}{70 a 79} & 1.9 & 0.52 \\
\hline & $(0.67-5.33)$ & $(0.23-1.17)$ \\
\hline \multirow[t]{2}{*}{80 o más } & 1.16 & 0.46 \\
\hline & $(0.41-3.21)$ & $(0.21-1.02)$ \\
\hline \multicolumn{3}{|c|}{ Número de enfermedades en el UAV (0 ref.) } \\
\hline \multirow[t]{2}{*}{1} & $1.77 * *$ & $1.61^{*}$ \\
\hline & $(1.22-2.57)$ & $(1.14-2.26)$ \\
\hline \multirow[t]{2}{*}{2} & $2.42 * * *$ & $2.82 * * *$ \\
\hline & $(1.49-3.96)$ & $(1.71-4.65)$ \\
\hline \multirow[t]{2}{*}{3 o más } & $3.55^{*}$ & $4.34 *$ \\
\hline & $(1.15-10.96)$ & $(1.25-15.01)$ \\
\hline \multicolumn{3}{|c|}{ Con limitación (Sin limitación ref.) } \\
\hline \multirow[t]{2}{*}{ Con ABVD } & 1.44 & 1.14 \\
\hline & $(0.91-2.26)$ & $(0.73-1.77)$ \\
\hline \multirow[t]{2}{*}{ Con AIVD } & 1.37 & $1.85^{*}$ \\
\hline & $(0.86-2.15)$ & $(1.18-2.91)$ \\
\hline \multirow[t]{2}{*}{ Constante } & 0.61 & 1.67 \\
\hline & $(0.22-1.66)$ & $(0.74-3.71)$ \\
\hline
\end{tabular}

$* \mathrm{P}<0.05 ; * * \mathrm{P}<0.005 ; * * * \mathrm{P}<0.001$

Fuente: elaboración propia con base en ENASEM 2001-2015. 